View Article Online / Journal Homepage / Table of Contents for this issue

FORMATION AND STABILITY OF SPIRO-COMPOUNDS. PART VII. 513

\title{
LX.-The Formation and Stability of spiro-Compounds. Part VII. The Application of the Dieckmann Reaction to Esters of the Glutaric Series.
}

\section{By George Armand Robert Kon.}

Is Part IV of this series (Kon, T., 1921, 119, 810), it was pointed out that glutaric acid does not give rise to compounds of the cyclobutane series by Wislicenus's or Dieckmann's reactions, apparently because the acetic residues in this acid are too far apart to interact in the required manner. It was shown that by introducing a gem-grouping or ring into the chain in the $\beta$-position it was possible to cause a convergence of the acetic residues (loc. cit., p. 812), and that acids substituted in this way could be made to undergo Wislicenus's reaction, although the cyclic ketones primarily formed were found to isomerise to unsaturated ketones.

The tendency to pass into a cyclic ketone which a given acid should possess was expressed mathematically in the form of "formation numbers" by Dr. C. K. Ingold (see his note to the author's paper, loc. cit., p. 816), and it was shown that the acids studied formed a series connecting the glutaric with the adipic series of dicarboxylic acids as regards their tendency to pass into cyclic ketones.

An attempt was made to establish a "critical formation number " for the production of cyclic ketones by the Wislicenus reaction, and it was concluded that this figure lay somewhere in the region between the figures 0.43 (for glutaric acid) and 0.38 (for $\beta \beta$-dimethylglutaric acid); the critical "figure of instability," on the other 
hand, could not be ascertained because in all cases the cyclic ketone underwent isomerisation.

It appeared of great interest to study the application of another classical reaction to the same series of acids. This reaction is that elaborated by Dieckmann (Ber., 1894, 27, 102 ; Annalen, 1901, 317, 27 ) and consists in the elimination of alcohol from the ester of a suitable dibasic acid :

$$
\begin{aligned}
\left(\mathrm{CH}_{2}\right)_{n}<\mathrm{CH}_{2} \cdot \mathrm{CO}_{2} \mathrm{R} & \stackrel{\mathrm{Na}}{\longrightarrow} \\
\left(\mathrm{CH}_{2}\right)_{n} & \left.<\mathrm{CH}_{2}-\mathrm{CO}_{2} \mathrm{R}\right)
\end{aligned}
$$

The formation numbers of the ketonic esters are identical with those of the corresponding ketones (loc. cit., p. 818). The calculation of their figures of instability is attended with difficulty, but it is plain on general grounds that they must show the same mutual relationship as those given for the ketones (loc. cit., p. 817), and it therefore follows that of the esters investigated it is the cyclohexane compound which should be the most easily formed and the most stable. It may be stated at once that this conclusion is fully borne out by experiment.

The action of sodium on ethyl glutarate has already been studied by Dieckmann himself (Annalen, 1901, 317, 27), who succeeded in obtaining a very small amount of oil insoluble in aqueous sodium carbonate and giving a blue colour with alcoholic ferric chloride. $\mathrm{He}$ was unable to investigate it further or to obtain the expected cyclobutanone from it on hydrolysis.

Dieckmann's experiments were therefore repeated, and extended to the esters of three other acids, namely, $\beta \beta$-dimethylglutaric, cyclopentane-, and cyclohexane-diacetic acids, sodium and potassium being used as condensing agents under comparable conditions.

The difference in the behaviour of the last-named three esters from the first one was most marked.

Ethyl glutarate readily reacts with sodium or potassium, but on working up the product it is found that the substance formed consists entirely of an extremely viscous gum, completely soluble in aqueous sodium hydrogen carbonate, although insoluble both in ether and in water. So far all attempts to isolate a pure compound from this substance have failed.

The action of potassium (or sodium) on the other three esters, on the other hand, gives rise to definite and well-characterised products, and it will be shown later that their formation is originally due to the production of a cyclic ketonic ester. Such a ketonic ester has in no case been isolated, but in the experiments with ethyl 
cyclohexanediacetate it was possible to isolate the cyclic ketone derived from the ester by the loss (by hydrolysis) of a carbethoxyl group, namely cyclohexanespirocyclobutanone (I), the constitution of which is placed beyond doubt by its oxidation to cyclohexane-1carboxylic-1-acetic acid (II).

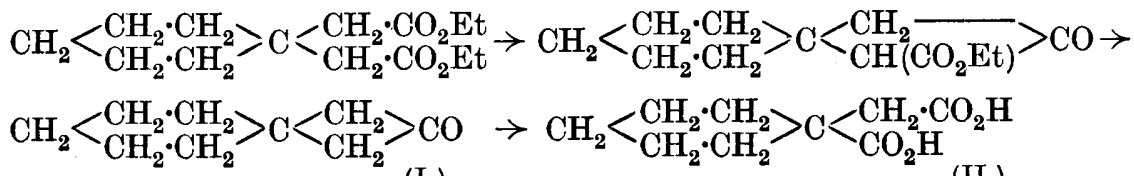

This remarkable substance is analogous to cyclohexanespirocyclopropanone, which was obtained in the form of its semicarbazone by Beesley, Ingold, and Thorpe (T., 1915, 107, 1080). The quantity prepared by them was, unfortunately, too small to allow the isolation of the ketone itself.

The yield of the new ketone is also very poor, barely reaching 1 per cent. of the theoretical, and it has therefore only been possible to study the most important properties of the substance.

The ketone is saturated, being quite stable to neutral permanganate, and is only slowly attacked by the alkaline reagent in the cold.

The optical properties of the substance were determined, and it was found that there was a marked exaltation in the molecular refraction $(+0 \cdot 77)$ and also in the dispersion. A similar exaltation due to the presence of the cyclobutane ring is observed in the isomeric nopinone (III) (+0.57) (Oestling, T., 1912, 101, 457); the increase is not, however, as great as in the case of sabina ketone (IV), which contains a cyclopropane ring $(+0.96)$ (Wallach, Annalen, 1907, $359,265)$.

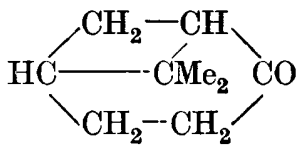

(III.)

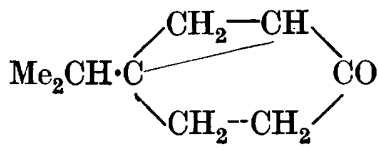

(IV.)

In addition to the ketone (I) and unchanged cyclohexanediacetic ester, as well as cyclohexanediacetic acid resulting from the hydrolysis of the ester, a solid diketone, $\mathrm{C}_{10} \mathrm{H}_{14} \mathrm{O}_{2}$, is formed in considerable amount. This substance is acid in character, and gives a deep violet coloration with alcoholic ferric chloride, which at first led to the belief that it was'a $1: 3$-diketone, but further investigation showed that the ketone groups were in the $-1: 2$-position to one another. Like all $1: 2$-diketones, the substance is quantitatively 
oxidised by alkaline hydrogen peroxide, the product being cyclohexane-1 : 1-diacetic acid. From this follows the constitution of the diketone, which must be cyclohexanespirocyclopentane-3 :4-dione (V).

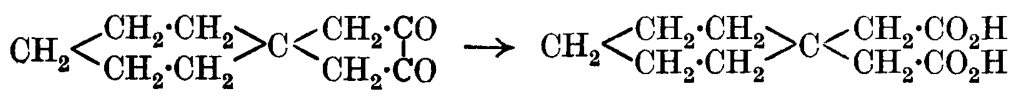

The parent member of this group of diketones, cyclopentane1:2-dione (VI), has already been studied by Dieckmann (Ber., $1902,35,3201)$, who showed that it is a tautomeric substance of the keto-enol type and reacts in accordance with the formula (VII)

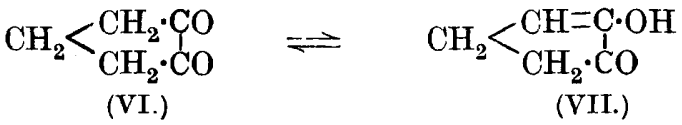

when it is treated with reagents for the hydroxyl group. The cyclohexane compound appears to be analogous in every way, and has been characterised by the formation of derivatives.

The formation of the diketone can only be explained by assuming that the original cyclobutane-ester undergoes fission by reduction in the strongly reducing medium in which it is formed, with the production of an aldehyde-ester from which alcohol is immediately eliminated with the formation of a diketone :

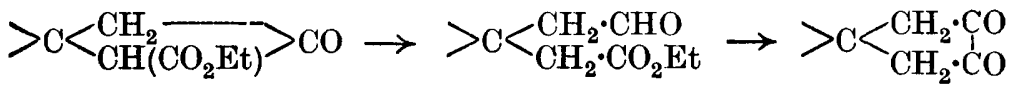

The process thus somewhat resembles the formation of benzoin from two molecules of benzaldehyde. It is, however, distinctly unusual, and experiments have been undertaken in order to shed further light on the subject. It is hoped that by using other condensing agents such as sodamide or sodium ethoxide, ring closure may be effected in a medium not tending to reduce the cyclic compounds formed.

When $\beta \beta$-dimethylglutaric ester was subjected to the action of sodium or potassium, the course of the reaction was found to be similar, but with important differences. In the first place, no trace of 1:1-dimethylcyclobutanone could be detected amongst the neutral products of the reaction, in spite of the most exhaustive search. In the second place, the yield of enolic material was very much smaller, amounting only to some $12-15$ per cent. of the theoretical. There can be little doubt that these differences are due to the smaller tendency on the part of $\beta \beta$-dimethylglutaric ester to pass into the ketonic ester from which the other compounds are 
derived, and to the greater instability of the cyclobutane ring in this case; this is entirely in accordance with the predictions based on Beesley, Ingold, and Thorpe's hypothesis.

The diketone formed from $\beta \beta$-dimethylglutaric ester is in every way analogous to the cyclohexane compound described above; it is identical with $1: 1$-dimethylcyclopentane-3:4-dione, which has already been obtained by Thorpe (T., 1911, 99, 2010) from methyl diketoapocamphorate.

It was thought necessary to make certain that the possible formation of 1:1-dimethylcyclobutanone was not overlooked on account of its boiling point, which should not be very different from that of xylene, which is used as solvent in this reaction, and would render its separation a matter of difficulty. It was therefore decided to investigate the behaviour of cyclopentanediacetic ester towards potassium.

Now the formation numbers for ketones (or ketonic esters) from this ester and $\beta \beta$-dimethylglutaric ester are almost identical, but the spiroketone should boil at a considerably higher temperature and should be isolable without difficulty. It is therefore very significant that no trace of such a compound was found in the neutral portion of the reaction mixture. A semicarbazone was isolated from this fraction, but analysis showed that it has the formula $\mathrm{C}_{10} \mathrm{H}_{17} \mathrm{O}_{2} \mathrm{~N}_{3}$, corresponding with a ketone, $\mathrm{C}_{9} \mathrm{H}_{14} \mathrm{O}_{2}$ (IX), and this was doubtless derived from the diketone (VIII) by reduction, thus:

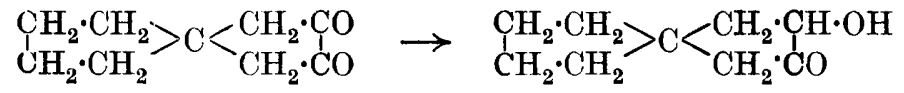

(VIII.)
(IX.)

cycloPentanespirocyclopentane-3:4-dione (VIII) was isolated from the oil insoluble in sodium hydrogen carbonate solution but soluble in aqueous potassium hydroxide. The yield of this substance was larger than that of the corresponding dimethyl compound, but smaller than that obtained from cyclohexanediacetic ester. The new diketone is in every way analogous to its higher homologue, and has been characterised by the formation of derivatives.

From the above results it is clear that the cyclobutane ring is only able to survive the experimental conditions of the Dieckmann reaction when it is combined with a cyclohexane ring, as shown by the fact that cyclohexanespirocyclobutanone is the only cyclobutane compound isolated in the present investigation; in the other cases the fission of the cyclobutane ring with the subsequent formation of a cyclobutane ring proceeds to completion. This result is clearly in exact agreement with the theoretical conclusion expressed above. 
With regard to the critical formation number, it is felt that definite conclusions cannot be drawn at present. The yields of material traceable to the formation of a cyclobutane ring, in the three cases where it has been demonstrated, clearly accord with the predictions based on theoretical figures; but the fact that no such compounds were obtained from glutaric ester is very probably due to another cause altogether. The product, in this case, is obviously of high molecular weight and may be derived from the interaction of two or more molecules of glutaric ester. Such a reaction may depend on the fact that the $\beta$-carbon atom is not substituted and can participate in the condensation, whilst this is clearly impossible when the $\beta$-carbon atom is quaternary. Further experiments will therefore be necessary to elucidate the point.

\section{EX P E R M E N T A L.}

The method used in carrying out the experiments with sodium is as follows.

Sodium (9 grams) is melted under dry xylene ( 50 c.c.) and pulverised by violent shaking. The ester $(0.2$ gram-mol.) is added to the hot mixture, and the flask, provided with a short air-condenser, is heated in an oil-bath maintained at about $150^{\circ}$ for three-quarters of an hour. The sodium gradually dissolves and a brown or red jelly fills the flask. Frequent shaking is necessary to prevent local overheating, leading to decomposition, and it is necessary to add another 25 c.c. of xylene after twenty to thirty minutes to prevent the mass from becoming too solid. When cold, the reaction mixture is decomposed with ice and ice-cold dilute sulphuric acid, which are added with constant shaking; the products of five such operations are combined. The acid aqueous layer is separated from the xylene solution and extracted once with ether, which is then added to the xylene solution. After washing once with water, this solution is thoroughly extracted with aqueous sodium hydrogen carbonate (extract $A$ ), then twice with ice-cold 15 per cent. aqueous potassium hydroxide (extract $B$ ).

After washing with water and drying over anhydrous sodium sulphate, the ether-xylene solution can be fractionated with a column; it contains the neutral products of the reaction (mainly unchanged starting-material).

Extract $A$ yields on acidification a certain quantity of the original substituted glutaric acid, which can be recovered; it contains practically none of the diketone.

Extract B, or "quasi-acid" fraction, is freed from traces of neutral substances by extraction with ether, and acidified with 
hydrochloric acid, a rise of temperature being prevented by adding ice to the solution. The precipitated oil, consisting of a diketone and the acid-ester of the corresponding glutaric acid in about equal proportions, is taken up in ether, the solution washed with aqueous sodium hydrogen carbonate, dried, and evaporated. The diketone can then be isolated by distillation under reduced pressure or by conversion into the semicarbazone.

The condensation with potassium is carried out in the same manner, but owing to the very vigorous reaction which takes place it is necessary to allow the "molecular" potassium, to which the requisite additional amount of xylene has been added, to cool completely before the ester is added. The reaction takes place without heating and may become very violent. Vigorous shaking is necessary at this stage, and a fairly long air-condenser should be used to prevent loss. The whole operation occupies a few minutes only. An alternative method consists in gradually adding the ester to the hot potassium with constant shaking. The method of working up is the same as with sodium.

There appears to be but little difference in the yields obtained by the two methods, with a slight balance in favour of potassium. The latter is, however, very much more convenient to use, as the long and tedious process of heating the reaction mixture becomes unnecessary.

\section{(I) Ethyl Glutarate.}

Potassium was used in these experiments. This ester reacts completely, but the product is totally different in character from that produced from the other esters investigated.

In the first place, on adding ice and dilute sulphuric acid to the reaction product it is found that an almost black, gummy material is precipitated, which is almost insoluble both in the aqueous and the xylene-ether layers. It is, however, completely soluble in aqueous sodium hydrogen carbonate, and it is found that, after treatment with the latter, the ethereal solution consists entirely of ether and xylene, and leaves almost no residue on distillation.

The sodium hydrogen carbonate solution, on acidification, yields the gum unchanged, and so far all attempts to isolate a pure substance from this have been fruitless, as it could not be induced to solidify and decomposed when distilled under reduced pressure (12 mm.); it gave an intense colour with alcoholic ferric chloride. An attempt was made to esterify it with alcohol and sulphuric acid in the cold in the hope of obtaining an ester (or ether) which could be distilled. A small amount of distillate was actually obtained, boiling at about $210^{\circ} / 12 \mathrm{~mm}$. and still giving a colour with ferric 
chloride, but the analysis gave no clue to its constitution (Found : $\mathrm{C}=65.82 ; \mathrm{H}=8.12$ per cent.) ; the high boiling point indicates beyond doubt that it has a high molecular weight. The substance is probably produced by the interaction of two or more molecules of glutaric ester, and cannot be analogous to the ketonic compounds obtained from other esters of this series.

The presence of cyclobutanone in the neutral fraction could not be demonstrated in spite of a careful search.

\section{(II) Ethyl cycloHexane-1 : 1-diacetate.}

The yields obtained from 1 gram-mol. of this ester with potassium were usually cyclohexanediacetic acid, and its ester, 35 grams and 50 grams, respectively, and quasi-acid fraction (crude), 70-75 grams.

When the condensations were carried out with sodium, it was found that some of the sodium was left unchanged, and the quantity of sodium had, therefore, to be reduced (from 9 to 7 grams for 0.2 gram-mol.). The yields of quasi-acid fraction were correspondingly small.

Isolation of cycloHexanespirocyclobutane-3-one (I).-This ketone occurs in varying, although always small, amounts in the neutral portion of the reaction product. The fraction boiling between $160^{\circ}$ and $250^{\circ}$ is treated with semicarbazide acetate in alcoholic solution and allowed to stand. The mixture is diluted with water after twenty-four hours, the dilution usually causing the separation of a certain amount of xylene. As the semicarbazone is appreciably soluble in xylene, light petroleum (b. p. $40-60^{\circ}$ ) is added at this stage to prevent loss from this cause. The yield of semicarbazone obtained in this way rarely exceeds 1 gram, and in a few experiments much less was obtained. The best yield is about 1 per cent. of the theoretical.

The semicarbazone crystallises from dilute alcohol in small needles melting at $204^{\circ}$ (Found: $\mathrm{C}=61.46 ; \mathrm{H}=8.82 ; \mathrm{N}=21 \cdot 79$. $\mathrm{C}_{10} \mathrm{H}_{17} \mathrm{ON}_{3}$ requires $\mathrm{C}=61.51 ; \mathrm{H}=8.77 ; \mathrm{N}=21.53$ per cent.).

The ketone was regenerated from the semicarbazone by boiling with aqueous oxalic acid in a current of steam, and was isolated from the distillate by means of ether. It is a perfectly colourless, mobile liquid, with a characteristic camphoraceous odour, and boils at $208^{\circ} / 751 \mathrm{~mm}$. (thermometer in vapour up to $100^{\circ}$; pure naphthalene boiled at $213.5^{\circ}$ under the same conditions) (Found : $\mathrm{C}=78 \cdot 06 ; \mathrm{H}=10 \cdot 12 . \quad \mathrm{C}_{9} \mathrm{H}_{14} \mathrm{O}$ requires $\mathrm{C}=78 \cdot 20 ; \quad \mathrm{H}=10 \cdot 21$ per cent.).

Soon after distillation the density was found to be 0.96105 at $20.7^{\circ}$. Two days later it was 0.96220 , and the refractive indices 
determined at the same time were: $n_{\mathrm{c}}=1.46858 ; n_{\mathrm{D}}=1.47113$; $n_{\mathrm{F}}=1.47736 ; n_{\mathrm{G}}=1.48447$. Hence $\left[\mathrm{R}_{\mathrm{L}}\right]_{\mathrm{D}}=40 \cdot 14 ;\left[\mathrm{R}_{\mathrm{L}}\right]_{\mathrm{G}-\mathrm{C}}=1 \cdot 156$, and $\left[\mathrm{R}_{\mathrm{L}}\right]_{\mathrm{F}-\mathrm{C}}=0.64$, the calculated values being $39 \cdot 37,0.99$, and 0.60 respectively. All the values thus show considerable exaltation, comparable with those found by Oestling (loc. cit.) for nopinone and by Wallach for sabina ketone.

The stability of the ketone towards 1 per cent. aqueous permanganate is considerable, the neutral reagent being scarcely affected for some hours; in the presence of sodium carbonate reduction takes place in about three-quarters of an hour. When boiled in alcoholic solution with $p$-nitrophenylhydrazine, the ketone readily gives a p-nitrophenylhydrazone, forming stout, orange needles melting at $137-138^{\circ}$ after two crystallisations from dilute alcohol (Found : $\mathrm{N}=15 \cdot 70 . \quad \mathrm{C}_{15} \mathrm{H}_{19} \mathrm{O}_{2} \mathrm{~N}_{3}$ requires $\mathrm{N}=15 \cdot 37$ per cent.).

The constitution of the ketone was confirmed by oxidation. When the ketone was dropped into hot dilute nitric acid $(d 1 \cdot 2)$, the action was sluggish, and a little concentrated acid was therefore added. The action then proceeded briskly and was complete in a few minutes. On evaporating the acid liquor, an oily residue was obtained, which soon solidified on scratching and formed feathery aggregates of prisms melting at $132^{\circ}$ after two crystallisations from dilute hydrochloric acid. The substance produced in this way was found to be identical with cyclohexane-1-carboxylic. 1-acetic acid (II) by direct comparison (mixed melting point) with Norris and Thorpe's specimen* (T., 1921, 119, 1199) and by analysis (Found: $\mathrm{C}=5 \mathbf{5 7} \cdot 84 ; \mathrm{H}=7.52$. Calc., $\mathrm{C}=58.04$; $\mathrm{H}=7.58$ per cent.).

Examination of the Quasi-acid Fraction.-The enolic oil obtained from the extract $B$ as described on p. 518 boils for the most part between $160^{\circ}$ and $175^{\circ} / 20 \mathrm{~mm}$., although a considerable fraction is obtained in the neighbourhood of $200^{\circ} / 20 \mathrm{~mm}$. The lower fraction, on cooling, partly solidifies to a mass of colourless needles, which can be isolated by draining on porous porcelain; the pure substance boils at $158-159^{\circ} / 11 \mathrm{~mm}$.; the yield is $20-25$ per cent. of the theoretical.

The substance can be recrystallised from a small amount of light petroleum (b. p. $40-50^{\circ}$ ); it is extremely soluble in other organic solvents, but almost insoluble in water. The compound separates from a concentrated solution in light petroleum in long needles melting at $67^{\circ}$, but if it is allowed to separate slowly from a more dilute solution, it forms thick, transparent, twinned crystals,

* The melting point of this substance is given (loc. cit.) as $134^{\circ}$, but it was found to melt at $132^{\circ}$ with the same thermometer and under the same conditions as the above. 
belonging to a biaxial system. This compound is cyclohexanespirocyclopentane-3 : 4-dione (V) (Found : $\mathrm{C}=72.30 ; \mathrm{H}=8.68$. $\mathrm{C}_{10} \mathrm{H}_{14} \mathrm{O}_{2}$ requires $\mathrm{C}=72 \cdot 25 ; \mathrm{H}=8.49$ per cent.).

The diketone gives an intense violet coloration with ferric chloride in alcoholic solution. It is soluble in alkalis, forming a deep red solution, is only partly soluble in alkali carbonates, and does not effervesce with a solution of sodium hydrogen carbonate. With semicarbazide acetate in excess, it forms an extremely insoluble disemicarbazone, which melts and decomposes suddenly at $248^{\circ}$. This substance is sufficiently soluble in hot acetic acid to be recrystallised from that solvent, and separates in small, colourless prisms on cooling. It then melts and decomposes at $241^{\circ}$; the cause of the lowering of the melting point has not been ascertained. For most purposes, the compound is best purified by boiling with alcohol (Found : $\mathrm{C}=51 \cdot 29 ; \mathrm{H}=7 \cdot 27 ; \mathrm{N}=30 \cdot 15$. $\mathrm{C}_{12} \mathrm{H}_{20} \mathrm{O}_{2} \mathrm{~N}_{6}$ requires $\mathrm{C}=51.39 ; \mathrm{H}=7.21 ; \mathrm{N}=29.98$ per cent.).

The hydrolysis of this disemicarbazone presents considerable difficulties. Aqueous oxalic acid has but little action owing to the sparing solubility of the compound, whereas mineral acids, especially when moderately concentrated, lead to the formation of a compound, $\mathrm{C}_{11} \mathrm{H}_{15} \mathrm{ON}_{3}$, which resists further hydrolysis. Five to 10 per cent. aqueous sulphuric acid is the best hydrolytic agent found up to the present and allows a fair yield of the pure diketone to be obtained from the disemicarbazone. The diketone can then be isolated by distillation in a current of steam and extraction of the distillate with ether.

As already mentioned, the use of concentrated mineral acids leads to the production of a compound, $\mathrm{C}_{11} \mathrm{H}_{15} \mathrm{ON}_{3}$, which is always formed to a certain extent in the hydrolysis of the disemicarbazone and crystallises out on cooling the acid mixture. There is little doubt that this compound is analogous to the triazine obtained by Thiele and Stange (Annalen, 1894, 283, 27) from benzil and semicarbazide, and has the constitution

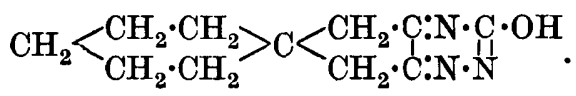

It forms buff needles when crystallised from acetic acid, and decomposes at $295^{\circ}$; it is very sparingly soluble in other organio solvents (Found : $\mathrm{C}=64.47 ; \mathrm{H}=7 \cdot 32 ; \mathrm{N}=20.66$. $\quad \mathrm{C}_{11} \mathrm{H}_{15} \mathrm{ON}_{3}$ requires $\mathrm{C}=64.36 ; \mathrm{H}=7.35 ; \mathrm{N}=20.47$ per cent.). The compound is soluble in sodium hydroxide solution and dissolves with a deep orange colour in concentrated sulphuric acid.

With $p$-nitrophenylhydrazine the diketone forms a beautifully scarlet osazone, which decomposes at $275^{\circ}$ and is very sparingly 
soluble in most organic solvents. It gives an intense crimson coloration with concentrated sulphuric acid (Found : $\mathrm{C}=60.63$; $\mathrm{H}=5.76 . \quad \mathrm{C}_{22} \mathrm{H}_{24} \mathrm{O}_{4} \mathrm{~N}_{6}$ requires $\mathrm{C}=60.51 ; \mathrm{H}=5.55$ per cent.).

o-Phenylenediamine readily condenses when warmed with the diketone in alcoholic solution for a few minutes. The quinoxaline derivative, $\mathrm{C}_{5} \mathrm{H}_{10}>\mathrm{C}<\frac{\mathrm{CH}_{2} \cdot \mathrm{C}: \mathrm{N}}{\mathrm{CH}_{2} \cdot \mathrm{C}: \mathrm{N}}>\mathrm{C}_{6} \mathrm{H}_{4}$, crystallises from dilute alcohol in beautiful, silky, flattened needles melting at $99-100^{\circ}$ and retaining a buff tint even on repeated crystallisation (Found : $\mathrm{C}=80 \cdot 46 ; \mathrm{H}=7 \cdot 77 . \quad \mathrm{C}_{16} \mathrm{H}_{18} \mathrm{~N}_{2}$ requires $\mathrm{C}=80 \cdot 63 ; \mathrm{N}=7 \cdot 60$ per cent.).

An anil, $\mathrm{C}_{5} \mathrm{H}_{10}>\mathrm{C}<\underset{\mathrm{CH}_{2} \cdot \mathrm{C} \cdot \mathrm{NPh} \text {, }}{\mathrm{CH}=\mathrm{C} \cdot \mathrm{OH}}$ is also readily obtained and melts at $106^{\circ}$; it forms lustrous, colourless, needles from dilute alcohol (Found : $\mathrm{C}=79 \cdot 20 ; \quad \mathrm{H}=8.08 . \quad \mathrm{C}_{16} \mathrm{H}_{19} \mathrm{ON}$ requires $\mathrm{C}=79.60 ; \mathrm{H}=7.96$ per cent.).

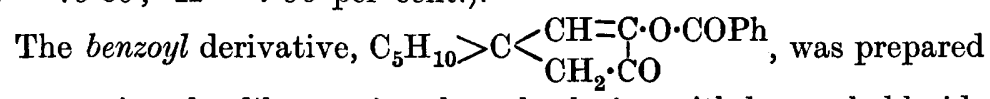
by warming the diketone in ethereal solution with benzoyl chloride and pyridine. It crystallises from light petroleum (b. p. 40-50 in spherical clusters of colourless needles melting at $69-70^{\circ}$ (Found: $\mathrm{C}=75.46 ; \mathrm{H}=6.97 . \mathrm{C}_{17} \mathrm{H}_{18} \mathrm{O}_{3}$ requires $\mathrm{C}=75.51$; $\mathrm{H}=6.70$ per cent.).

The constitution of the diketone was finally confirmed by oxidation. The diketone $(0.5$ gram) was dissolved in 10 c.c. of 10 per cent. aqueous sodium carbonate, and 25 c.c. of 6 per cent. hydrogen peroxide were added. A brisk evolution of gas soon occurred and the liquid became warm. It was acidified after about two hours, when a crystalline acid was precipitated; a further crop was obtained by extracting the mother-liquors with ether. The acid was identified as cyclohexane-1:1-diacetic acid by direct comparison (mixed melting point) and by analysis (Found : $\mathrm{C}=60.08$; $\mathrm{H}=8.08$. Calc., $\mathrm{C}=59.98 ; \mathrm{H}=8.05$ per cent.).

In addition to the diketone melting at $67^{\circ}$ there is another enolic substance which is present in small quantity in the original extract $B$. It is found in the fraction of higher boiling point (about $180^{\circ} / 20 \mathrm{~mm}$.) and partly separates in the solid state. The solid can be crystallised from a mixture of ether and light petroleum (b. p. $40-50^{\circ}$ ) and is obtained in small, colourless plates melting at $135-136^{\circ}$. It develops an intense violet coloration with alcoholic ferric chloride; its composition corresponds with the formula $\mathrm{C}_{12} \mathrm{H}_{18} \mathrm{O}_{2}$ (Found : $\mathrm{C}=73.92 ; \quad \mathrm{H}=9 \cdot 33$. Calc., $\mathrm{C}=74 \cdot 20$; $\mathrm{H}=9 \cdot 33$ per cent.), but the constitution of the substance has not yet been elucidated. 
The less volatile oil obtained from the quasi-acid fraction consists of the acid-ester of cyclohexanediacetic acid, although a little of the diketone is still present, as shown by the somewhat high percentage of carbon found on analysis (Found: $\mathrm{C}=64 \cdot 13 ; \mathrm{H}=8 \cdot 76$. $\mathrm{C}_{12} \mathrm{H}_{20} \mathrm{O}_{4}$ requires $\mathrm{C}=63.12 ; \mathrm{H}=8.85$ per cent.). It yields cyclohexanediacetic acid on hydrolysis.

\section{(III) Ethyl $\beta \beta$-Dimethylglutarate.}

Potassium was almost exclusively used as a condensing agent. The yields were $\beta \beta$-dimethylglutaric acid * and its ester, 30 grams and 52 grams respectively, and quasi-acid fraction, 16 grams. In spite of the most searching investigation, the formation of 1 : 1-dimethylcyclobutane-2-one could not be demonstrated.

Examination of the Quasi-acid Fraction.-As far as could be ascertained, only one substance occurs in this fraction; it is best separated in the form of its sparingly soluble disemicarbazone, which is readily formed under the ordinary conditions. It is a microcrystalline powder decomposing at $257^{\circ}$ with previous darkening ; crystallisation from acetic acid lowers the decomposition point to $251^{\circ}$ (compare p. 522) (Found: $\mathrm{C}=45 \cdot 04 ; \mathrm{H}=6 \cdot 80 . \quad \mathrm{C}_{9} \mathrm{H}_{16} \mathrm{O}_{2} \mathrm{~N}_{6}$ requires $\mathrm{C}=44.98 ; \mathrm{N}=6.71$ per cent.).

This disemicarbazone is more readily hydrolysed than the corresponding cyclohexane compound, and a 60 per cent. yield of pure 1: 1-dimethylcyclopentane-3:4-dione is easily obtained from it by means of an excess of 10 per cent. aqueous sulphuric acid. The yield of diketone appears to be barely 8 per cent. of the theoretical. The diketone is isolated by distillation in a current of steam and can be extracted from the distillate, previously saturated with ammonium sulphate, by means of ether. On removing the solvent, the diketone usually remains in the superfused state, but it can be made to crystallise immediately on introducing a crystal of the solid. It crystallises beautifully from light petroleum (b. p. $40-50^{\circ}$ ) in large plates resembling mica and melting at $45^{\circ}$, identical with the substance obtained by Thorpe (loc. cit.) from methyl diketoapocamphorate by hydrolysis (mixed melting point) (Found : $\mathrm{C}=66.32$; $\mathrm{H}=7 \cdot 97$. Calc., $\mathrm{C}=66.61 ; \mathrm{H}=7.93$ per cent.). The diketone gives a deep purplish-red colour with ferric chloride and is appreciably soluble in water.

The quinoxaline derivative, $\mathrm{Me}_{2} \mathrm{C}<\underset{\mathrm{CH}_{2} \cdot \mathrm{C}: \mathrm{N}}{\mathrm{CH}_{2} \cdot \mathrm{C}: \mathrm{N}}>\mathrm{C}_{6} \mathrm{H}_{4}$, is readily formed by warming 0.3 gram of the diketone in alcoholic solution with an equal weight of $o$-phenylenediamine for a few minutes,

* Some of the acid is lost owing to its great solubility in water. 
the alcohol being allowed to evaporate off on the steam-bath. On scratching, the mass solidifies. The product, crystallised from dilute alcohol, forms magnificent, flattened needles, m. p. 123.5 (Found : $\mathrm{C}=78.81 ; \mathrm{H}=7 \cdot 14 . \quad \mathrm{C}_{13} \mathrm{H}_{14} \mathrm{~N}_{2}$ requires $\mathrm{C}=78 \cdot 73$; $\mathrm{H}=7 \cdot 12$ per cent.).

The constitution of the diketone was confirmed by oxidation as in the case of the cyclohexane compound. The acid, obtained in quantitative yield by the action of hydrogen peroxide in alkaline solution, melted at $101^{\circ}$ and was proved to be $\beta \beta$-dimethylglutaric acid by direct comparison (mixed melting point) with a genuine specimen and by analysis (Found: $\mathrm{C}=52 \cdot 49 ; \mathrm{H}=7.52$. Calc., $\mathrm{C}=52 \cdot 47 ; \mathrm{H}=7 \cdot \dot{5} 6$ per cent.).

\section{(IV) Ethyl cycloPentanediacetate.}

This ester, prepared by esterifying the acid with alcohol and sulphuric acid, is a colourless liquid boiling at $157-158^{\circ} / 16 \mathrm{~mm}$. (Found: $\mathrm{C}=64 \cdot 29 ; \quad \mathrm{H}=9.09 . \quad \mathrm{C}_{13} \mathrm{H}_{22} \mathrm{O}_{4}$ requires $\mathrm{C}=64 \cdot 13$; $\mathrm{H}=9 \cdot 13$ per cent.).

The yields obtained from 1 gram-mol. of the ester, by interaction with potassium, were cyclopentanediacetic acid and its ester, 30 grams and 55 grams respectively, and the quasi-acid fraction (crude), 54 grams.

The neutral fraction was carefully searched for the presence of a ketone, $\mathrm{C}_{8} \mathrm{H}_{12} \mathrm{O}$, but this has not, up to the present, been detected. It was invariably found, however, that the acid diketone was not completely extracted by treatment with potassium hydroxide, and the neutral fraction still gave a faint coloration with ferric chloride. On prolonged standing with alcoholic semicarbazide acetate, a little of the disemicarbazone (decomp. $252^{\circ}$; see p. 526) was obtained. In addition to this, a semicarbazone soluble in alcohol was also produced. It was purified by crystallisation from dilute alcohol and formed stout, transparent crystals decomposing without darken. ing at $183-184^{\circ}$ (Found: $\mathrm{C}=56.43 ; \quad \mathrm{H}=8.20 ; \quad \mathrm{N}=20.22$. $\mathrm{C}_{10} \mathrm{H}_{17} \mathrm{O}_{2} \mathrm{~N}_{3}$ requires $\mathrm{C}=56.85 ; \mathrm{H}=8 \cdot 10 ; \mathrm{N}=19.94$ per cent.). It appears probable that the corresponding ketone has the constitu. tion IX.

Examination of the Quasi-acid Fraction.-This fraction consists of equal quantities of the acid ester* and the diketone VIII, which boils for the most part at $148^{\circ} / 13 \mathrm{~mm}$. and immediately solidifies on cooling; the yield is about 18 per cent. of the theoretical. It can be purified by crystallisation from light petroleum (b. p. $40-50^{\circ}$ ), in which it is somewhat sparingly soluble. From this

* This was confirmed by hydrolysis to cyclopentanediacetic acid. 


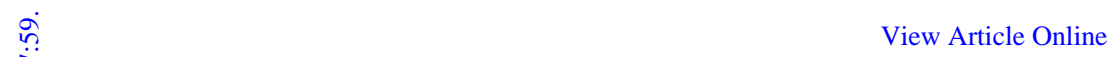

solvent it separates in fine, sparkling plates melting at $110-111^{\circ}$. The compound is cyclopentanespirocyclopentane-3 : 4-dione (Found : $\mathrm{C}=71.03 ; \quad \mathrm{H}=7.81 . \quad \mathrm{C}_{9} \mathrm{H}_{12} \mathrm{O}_{2}$ requires $\mathrm{C}=71.00 ; \quad \mathrm{H}=7.96$ per cent.). In alcoholic solution it gives an intense dark red colour with ferric chloride.

The disemicarbazone is a microcrystalline powder almost insoluble in alcohol, and decomposes at $252^{\circ}$ (Found : $\mathrm{C}=49 \cdot 16 ; \mathrm{H}=6.96$. $\mathrm{C}_{11} \mathrm{H}_{18} \mathrm{O}_{2} \mathrm{~N}_{6}$ requires $\mathrm{C}=49.60 ; \mathrm{H}=6.80$ per cent.)

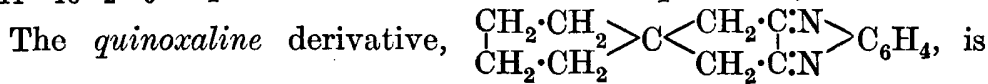
readily formed by the usual method, but is somewhat difficult to purify. It separates from dilute alcohol in buff needles and melts at $95-96^{\circ}$ (Found: $\mathrm{C}=79 \cdot 84 ; \mathrm{H}=7 \cdot 19 . \quad \mathrm{C}_{15} \mathrm{H}_{16} \mathrm{~N}_{2}$ requires $\mathrm{C}=80.32 ; \mathrm{H}=7 \cdot 19$ per cent.).

In conclusion, the author's best thanks are due to Professor J. F. Thorpe, F.R.S., for his kind interest in the progress of the investigation.

Imperial College of Sctence and Technology, South Kensington.

[Received, February 13th, 1922.]. 